\title{
Molecular Changes of Preclinical Scrapie Can Be Detected by Infrared Spectroscopy
}

\author{
Janina Kneipp, Michael Beekes, Peter Lasch, and Dieter Naumann \\ PG3, Robert Koch-Institut, D-13353 Berlin, Germany
}

Infrared (IR) microspectroscopy was used to detect diseaseassociated molecular changes spatially resolved in cryosections of scrapie-infected tissue of the CNS. The results show that IR spectra can be used for the discrimination between normal and 263K scrapie-infected hamster nervous tissue not only in the terminal stage of the disease but also in early clinical and even in the preclinical stage at $90 \mathrm{~d}$ after oral infection. The nuclei of the cranial nerves located in the medulla oblongata were especially well suited for an early detection of the dis- eased state by IR microspectroscopy. The most prominent molecular changes indicated by the IR spectra were located between 1300 and $1000 \mathrm{~cm}^{-1}$, a region that contains contributions primarily from carbohydrates and the phosphate backbones of nucleic acids but also from membrane constituents.

Key words: Fourier-transform infrared microspectroscopy; scrapie strain 263K; transmissible spongiform encephalopathy; spectral mapping; Syrian hamster; scrapie pathogenesis; medulla oblongata; cerebellum
Transmissible spongiform encephalopathies (TSE), such as scrapie in sheep and goats, bovine spongiform encephalopathy in cattle, and Creutzfeldt-Jakob disease in humans, are a family of fatal neurodegenerative disorders (Prusiner et al., 1998). Many aspects concerning this group of diseases are not yet understood and have been a matter of intense research. As was shown in a number of studies, histological and molecular differences in TSEaffected nervous tissue are manifold, reaching from deposition of pathological prion protein $\left(\mathrm{PrP}^{\mathrm{Sc}}\right)$ (Hedge et al., 1999) as the hallmark of the disease to changes in protein expression, changes in the composition of membrane systems (Choi et al., 1998), alterations in gene regulation (Riemer et al., 2000), and changes in processes such as apoptosis (Fairbairn et al., 1994) and loss of cell populations. The interplay of many different biochemical changes accounts for the TSE-specific pathology. Only a few methods can detect changes in many different biomolecules at the same time during one measurement, and even fewer can accomplish this in situ. Among those are the vibrational spectroscopic methods, such as infrared (IR) and Raman spectroscopy. The nature of an IR spectrum of tissue is that of a fingerprint or pattern, revealing specific biochemical information contained in all IR-active molecules. Spatially resolved IR spectroscopy [using microscopes coupled to Fourier-transform IR (FTIR) spectrometers] can be used to produce IR spectral maps that match with histological maps, because each tissue structure possesses a distinct biochemical composition (Lewis et al., 1996; Kidder et al., 1997; Wetzel and LeVine, 1999). Methods of spectral classification can be used to differentiate between various tissue types, using the multidimensional structural information based on the sum of molecules at a specific location in the tissue (Lasch and

\footnotetext{
Received Oct. 31, 2001; revised Dec. 27, 2001; accepted Jan. 7, 2002.

We thank Marion Joncic for the dissection of the brains, Hans Huser (Robert Koch-Institut, Berlin, Germany) for the kind gift of mAb 3F4, and Tricia McBride and coworkers (Institute for Animal Health, Neuropathogenesis Unit, Edinburgh, UK) for introducing one of us (J.K.) to PrP immunocytochemistry.

Correspondence should be addressed to Janina Kneipp or Dieter Naumann, P34, Robert Koch-Institut, Nordufer 20, D-13353 Berlin, Germany. E-mail: janina.kneipp@epost.de or naumannd@rki.de.

Copyright (C) 2002 Society for Neuroscience $0270-6474 / 02 / 222989-09 \$ 15.00 / 0$
}

Naumann, 1998), thereby adding biochemical information to known histological parameters. Here, we report on the use of FTIR microspectroscopy to study molecular alterations associated with TSE infection in sections of hamster brain from three anatomic regions: the dorsal motor nucleus of the vagus nerve (DMNV) with parts of the solitary tract nucleus (SolN), the nucleus of the hypoglossal nerve $(\mathrm{HypN})$, and the interposed cerebellar nucleus (IntN). The DMNV, followed by the SolN, was recently identified to be the first region showing deposition of pathological $\mathrm{PrP}^{\mathrm{Sc}}$ in hamsters orally challenged with scrapie (Beekes et al., 1998) and also in natural scrapie of sheep (van Keulen et al., 2000). To find out at which stage in the disease process spectral changes could be observed, we analyzed IR spectra at $90 \mathrm{~d}$ postinfection (d.p.i.), at 120 d.p.i., and in the terminal stage of orally transmitted scrapie in hamsters.

\section{MATERIALS AND METHODS}

Sample preparation and histology. All animal experiments were performed in accordance with European and German legal and ethical regulations. Twelve outbred Syrian hamsters (females) were challenged orally with $1-3 \times 10^{7} 50 \%$ intracerebral lethal doses $\left(\mathrm{LD}_{50 \mathrm{icc}}\right)$, corresponding to $1-3 \times 10^{2} 50 \%$ oral lethal doses $\left(\mathrm{LD}_{50 \text { p.o. }}\right)$ of scrapie strain $263 \mathrm{~K}$ as described previously (McBride et al., 2001). Twelve mockinfected hamsters of the same age were similarly fed normal brain homogenate and served as controls. At three time points, 90 d.p.i., 120 d.p.i., and in the terminal stage of the disease (150-160 d.p.i., varying between individuals), four infected and four control animals were killed with $\mathrm{CO}_{2}$. Brains were frozen and stored at $-70^{\circ} \mathrm{C}$. Cryosections were cut coronally, starting from the medulla oblongata. Two series, each containing five adjacent sections, were taken (Fig. 1). The plane of the first sequence (referred to as plane 1 in the following text) contained the HypN, the DMNV, and parts of the SolN, whereas a second sequence (plane 2) was cut through the cerebellar nuclei (Fig. 1). In each of the planes, the first and third sections $(10 \mu \mathrm{m}$ in thickness) were stained with $0.1 \%$ cresyl fast violet and $0.2 \%$ methylene blue, respectively. All second sections $(10 \mu \mathrm{m})$ were thaw-mounted on $1 \mathrm{~mm} \mathrm{BaF}_{2}$ windows for FTIR microspectroscopy. For confirmation of scrapie pathology, all fourth and fifth sections $(8 \mu \mathrm{m}$ each) were stained for the prion protein with monoclonal antibody (mAb) 3F4 (Kascsak et al., 1987) from cell culture $(1.4 \mu \mathrm{g} / \mathrm{ml} ; 1: 100)$ (a kind gift from Dr. Hans Huser, Robert KochInstitut, Berlin, Germany) and normal mouse serum (Dako Diagnostika, Hamburg, Germany) as controls, respectively, using a procedure adapted from Taraboulos et al. (1992). Biotinylated goat anti-mouse antibody 


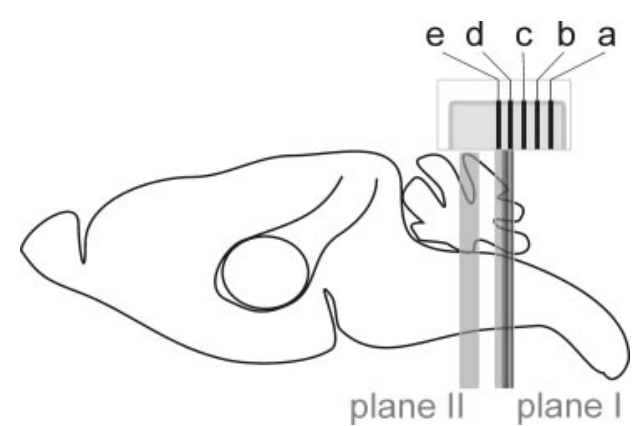

Figure 1. Coronal planes (schematic) investigated in this study. Plane 1 contained the hypoglossal nucleus, the DMNV, and parts of the SolN; plane 2 contained the cerebellar nuclei. The letters indicate the usage of the sections: $a$, Cresyl fast violet staining; $b$, FTIR microspectroscopy; $c$, methylene blue staining; $d, \mathrm{mAb} 3 \mathrm{~F} 4$ immunostaining; and $e$, normal mouse serum immunostaining (control).

(Dako Diagnostika), peroxidase-conjugated Vectastain avidin-biotin complex kit (Vector Laboratories, Burlingame, CA), and 3,3'diaminobenzidine as a substrate were used for detection of bound $\mathrm{mAb}$ 3F4. The immunostained sections were counterstained with hematoxylin.

Data acquisition and processing. An IFS28/B FTIR spectrometer (Bruker, Ettingen, Germany) coupled to an IR microscope A 590 (Bruker) equipped with a mercury-cadmium-telluride detector, circular apertures, a $15 \times$ Cassegrain objective, and a motorized stage (permitting the collection of spectra from a predefined grid of spots in a sample) and the Software OPUS 3.01 (Bruker) were used to take spectra in transmission mode from the tissue sections mounted to $\mathrm{BaF}_{2}$. Absorbance spectra were acquired in the spectral range of $4000-700 \mathrm{~cm}^{-1}$ at $6 \mathrm{~cm}^{-1}$ spectral resolution applying Happ-Genzel apodization and a zero filling factor of 4, yielding approximately one data point per wavenumber. The number of coadded interferograms per spectrum was set to 64 for "overview mapping" of larger tissue areas $\left(4-6 \mathrm{~mm}^{2}\right)$ using $100 \mu \mathrm{m}$ aperture diameter and to 512 scans for detailed mapping measurements using an aperture diameter of $50 \mu \mathrm{m}$.

Spectra of poor quality showing too high an absorption of water vapor or peak intensities that were too low or too high were excluded from the data sets as described previously (Kneipp et al., 2000). Chemical maps were reconstructed using an OPUS 3.01 macro to calculate a so-called "protein/lipid ratio" (integrated intensity at $1700-1480 \mathrm{~cm}^{-1}$, standing for the total protein content, divided by the integrated intensity at $3000-2838 \mathrm{~cm}^{-1}$, which reflects the total lipid content) for each spectrum of a data set and an in-house program to reassemble matrices necessary for the construction of IR maps in Origin 6.1 (OriginLab, Northampton, MA). For additional analyses, first derivatives of the spectra were calculated and subsequently vector normalized over the investigated frequency ranges. Hierarchical cluster analysis was performed on firstderivative spectra using the cluster analysis module of OPUS 3.01. It was applied to distinguish between different brain structure spectra from healthy and diseased brains in the terminal stage of scrapie using a frequency range between 1480 and $950 \mathrm{~cm}^{-1}$ and also to analyze normal and diseased tissue in earlier scrapie stages using the wavenumber range $1300-1000 \mathrm{~cm}^{-1}$. As input data for cluster analyses, spectral distances were calculated between pairs of spectra as Pearson's correlation coefficients as described by Helm et al. (1991). Cluster analyses for separation of diseased and control tissue were based on the Euclidean distances. In all cases, Ward's algorithm was used for hierarchical clustering. Spectral classes corresponding to specific brain structures were identified using cluster analysis-based maps, which were reconstructed by assigning a value on the gray scale to each spectrum in one spectral class. The combination of these gray-scale values with the original spatial information of each spectrum yielded the maps. Cluster analysis-based imaging was done with the CytoSpec program for IR imaging (www.cytospec. com). The spectra of these structure-specific classes were extracted and used for additional analyses, such as averaging, comparison of spectra from normal and diseased tissue by cluster analysis, and artificial neural network (ANN) analysis.

ANN analysis was performed on vector-normalized first derivatives with Synthon NeuroDeveloper 2.1 (Synthon, Gusterath, Germany). The windows $3050-2800 \mathrm{~cm}^{-1}$ and $1500-950 \mathrm{~cm}^{-1}$ were used for selection of significantly differing data points (with averaging over each two neigh-

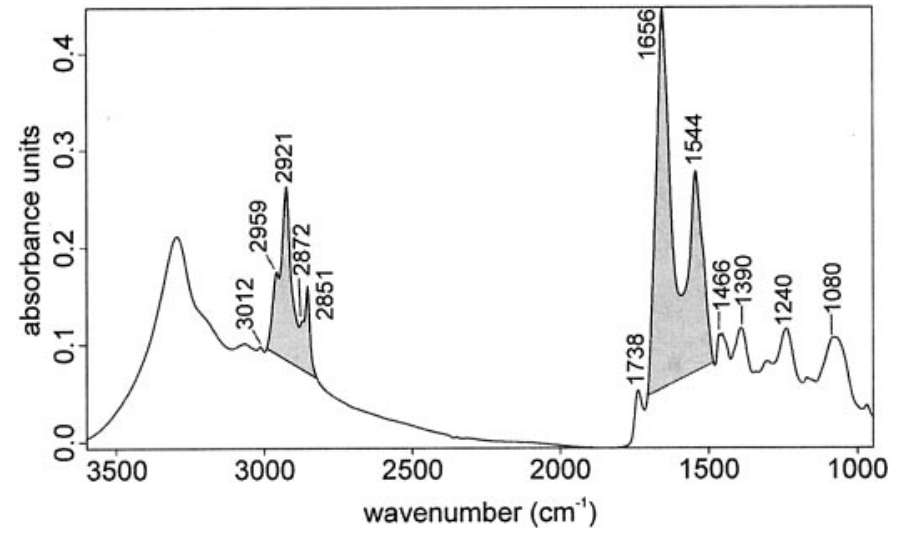

Figure 2. Typical IR spectrum obtained from the DMNV region of a hamster brain section. Major absorption bands are indicated (see also Table 1). The shaded regions are the amide region (integrated intensity at $1700-1480 \mathrm{~cm}^{-1}$ ) and the absorption bands of $\mathrm{CH}_{2}$ and $\mathrm{CH}_{3}$ groups (integrated intensity at $3000-2838 \mathrm{~cm}^{-1}$ ). Both regions were used to calculate the protein/lipid ratio used for IR imaging (Fig. 3). For more specific band assignments, see Table 1.

boring data points). Feature extraction was based on univariate $F$ values (Udelhoven et al., 2000). All data points with $F \geq 0.99$ were selected for network training, and the number of data points used for the networks at one disease stage was limited to the lowest number of data points of any of the three nuclei for comparative reasons. To avoid underdetermination of the system, the maximum number of input data points was limited to 100 . Fully connected, three-layer multilayer perceptrons (Goodacre, 2000) with the numbers of neurons in the input layer being the number of selected data points of the specific data set, 10 or 5 hidden neurons (number depending on the number of input neurons) and 2 output neurons (for the categories "infected" and "uninfected"), were trained using the $R_{\text {prop }}$ algorithm (Riedmiller and Braun, 1993). Training was stopped after a minimum sum squared error for the validation set was observed. The classification results were analyzed with the "winner takes all" function (Udelhoven et al., 2000). To ensure independent testing of all spectra from one nucleus and disease stage, test sets were assembled using the following procedure: All spectra of the data sets of control and infected tissue for one investigated nucleus of one disease stage (compare Table 2) were split into two parts, one comprising two-thirds and the other one-third of the total number of spectra. Each spectrum in these subsets was assigned a unique number starting from 1 . Then, beginning with spectrum 1 in each subset, every seventh spectrum of the subset was added to an independent test set. The test set contained spectra from both infected and control tissues. The remaining spectra of the two-thirds subset were used for training; those of the one-third subset were used for validation. After network training and classification of the spectra from the test data set, a new test set was constructed, this time beginning with the next spectrum in the subsets. This procedure was applied seven times. In this way, each pixel spectrum at one time point and nucleus was tested once as a member of the test group.

\section{RESULTS}

\section{IR spectral characteristics of brain tissue}

Figure 2 displays a typical IR spectrum of the DMNV. The information contained in such an IR absorption spectrum originates from all different types of biomolecules in the tissue, such as proteins, lipids, carbohydrates, and nucleic acids. The absorption bands result from the IR-active vibrations of the different functional groups contained in these molecules. Table 1 summarizes some important absorption bands of brain tissue IR spectra.

\section{Identification of brain structure-specific spectra}

IR images were reconstructed for the two coronal planes of interest in all 24 infected or control hamster brains. Plane 1 contained the DMNV/SolN and HypN, whereas plane 2 included the cerebellar nuclei. Figure 3 shows images for one brain sample 


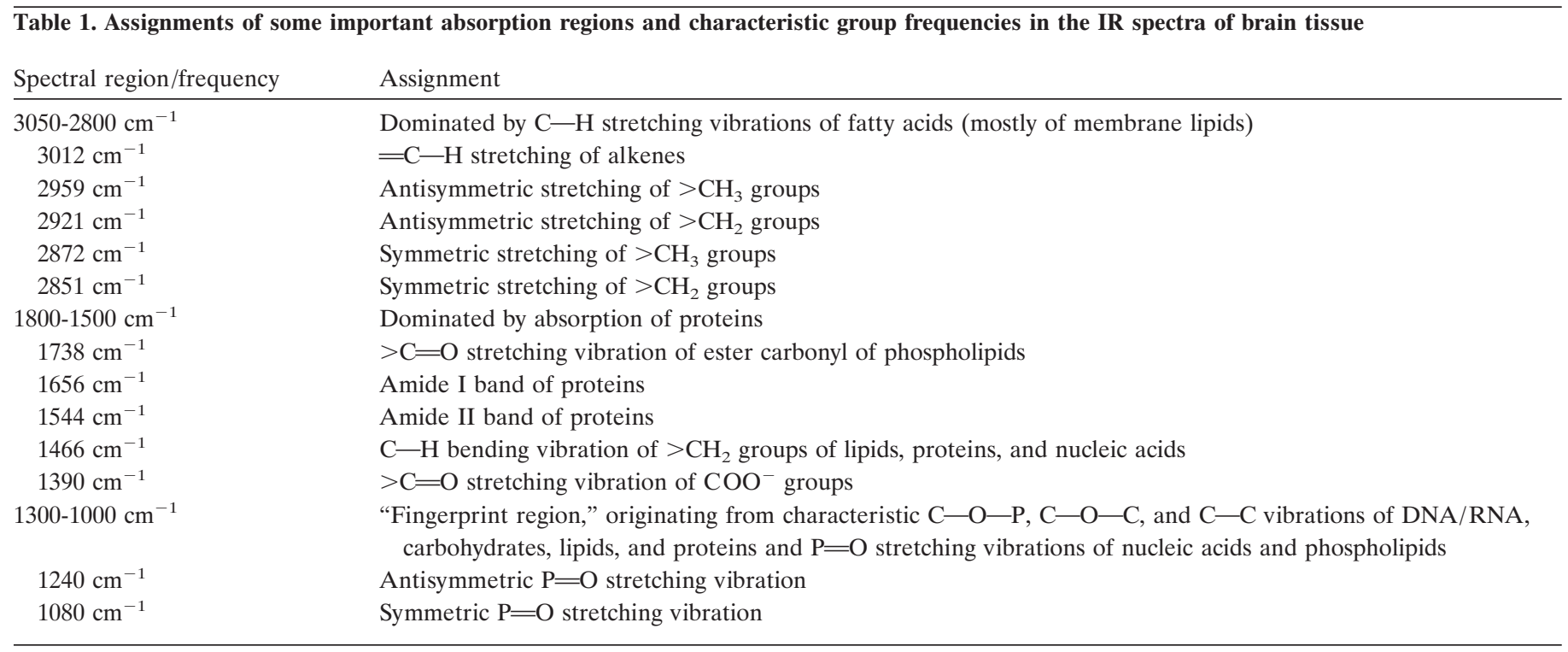

Dominated by $\mathrm{C}-\mathrm{H}$ stretching vibrations of fatty acids (mostly of membrane lipids)

Antisymmetric stretching of $>\mathrm{CH}_{2}$ groups

Symmetric stretching of $>\mathrm{CH}_{2}$ groups

Dominated by absorption of proteins

Amide I band of proteins

Amide II band of protein

$>\mathrm{C}=\mathrm{O}$ stretching vibration of $\mathrm{COO}^{-}$groups

"Fingerprint region," originating from characteristic $\mathrm{C}-\mathrm{O}-\mathrm{P}, \mathrm{C}-\mathrm{O}-\mathrm{C}$, and $\mathrm{C}-\mathrm{C}$ vibrations of DNA/RNA

Antisymmetric $\mathrm{P}=\mathrm{O}$ stretching vibration

Symmetric $\mathrm{P}=\mathrm{O}$ stretching vibration

A
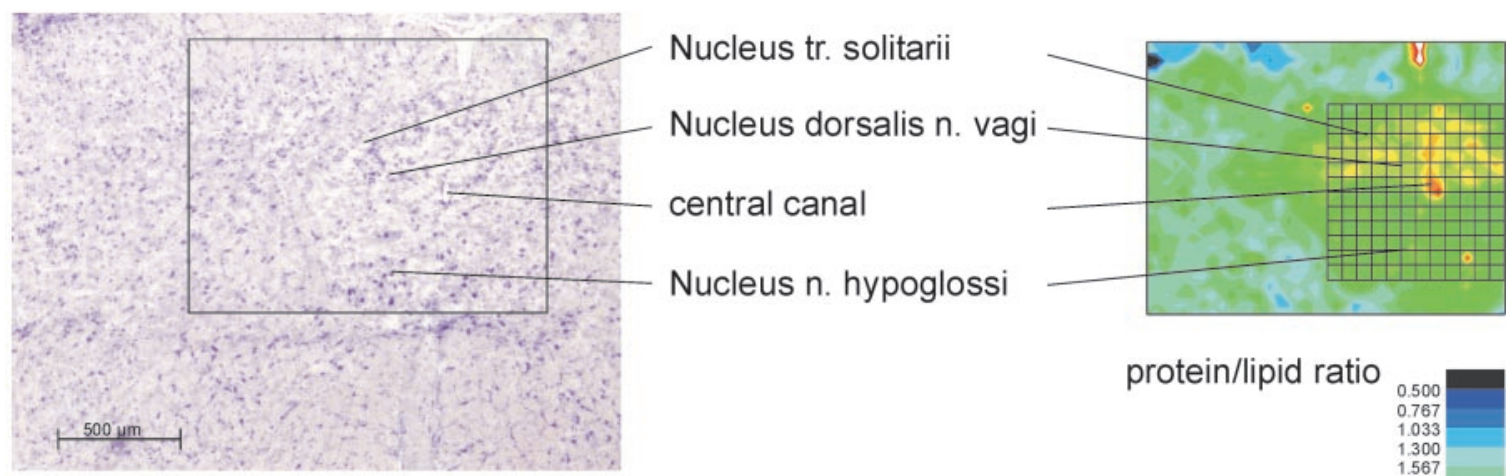

B

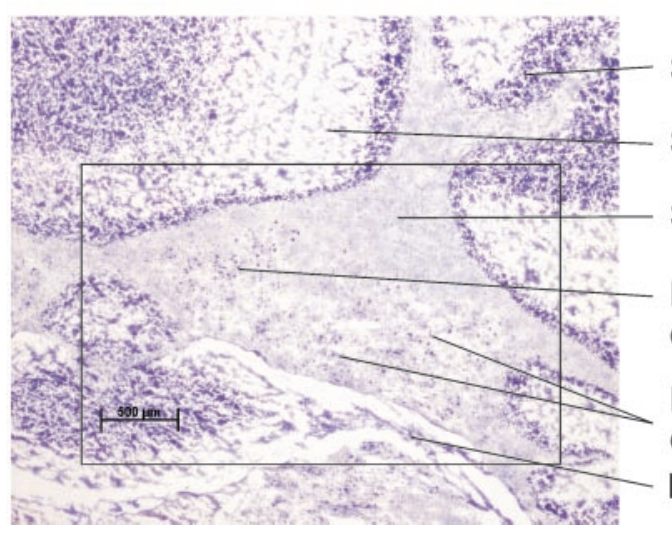

Stratum granulosum

Stratum moleculare

Substantia alba

Nucleus medialis cerebelli

Nucleus interpositus

cerebelli

Ependyma

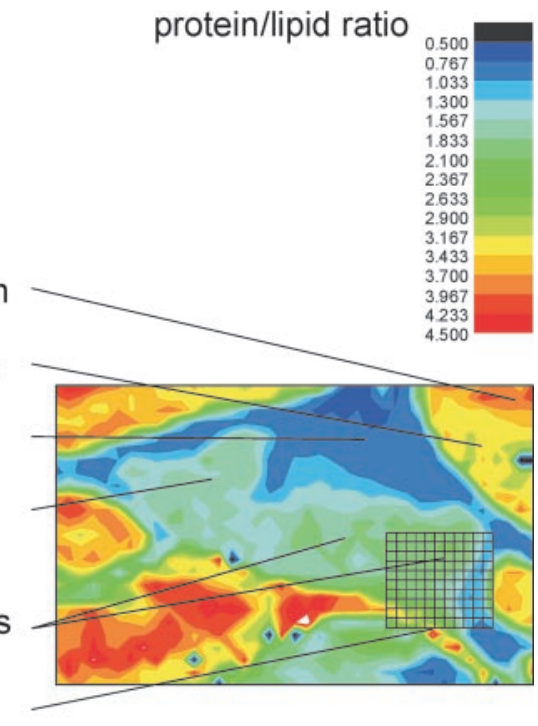

Figure 3. IR overview maps based on the protein/lipid ratio in the IR spectra (right) and cresyl fast violet stains of the adjacent tissue sections (left; scale bars, $500 \mu \mathrm{m}$ ) for orientation. Data obtained from one individual are shown as an example. $A$, Plane 1, containing the nuclei of the solitary tract, the DMNV, and the nucleus of the HypN. B, Plane 2, comprising the cerebellar nuclei and all cerebellar layers. The nuclei in the medulla oblongata $(A)$ and the cerebellum $(B)$ can be distinguished from the surrounding white matter by their high protein content. The insets in the photomicrographs (left) show the tissue area investigated by IR mapping. The grids in the IR maps indicate areas of detailed measurements that were performed for additional investigations.

as an example. They were reassembled using the ratio of the integral intensity of the amide bands and of the $\mathrm{CH}$-stretching region of lipids (both indicated in Fig. 2). The nuclei are relatively rich in protein content and therefore can be easily distinguished from the surrounding lipid-rich white matter in the overview images. After confirmation of the position of the nuclei in the IR maps by comparison with the adjacent cresyl fast violet-stained sections and with a brain atlas (Knigge and Joseph, 1968; Frank- 
A
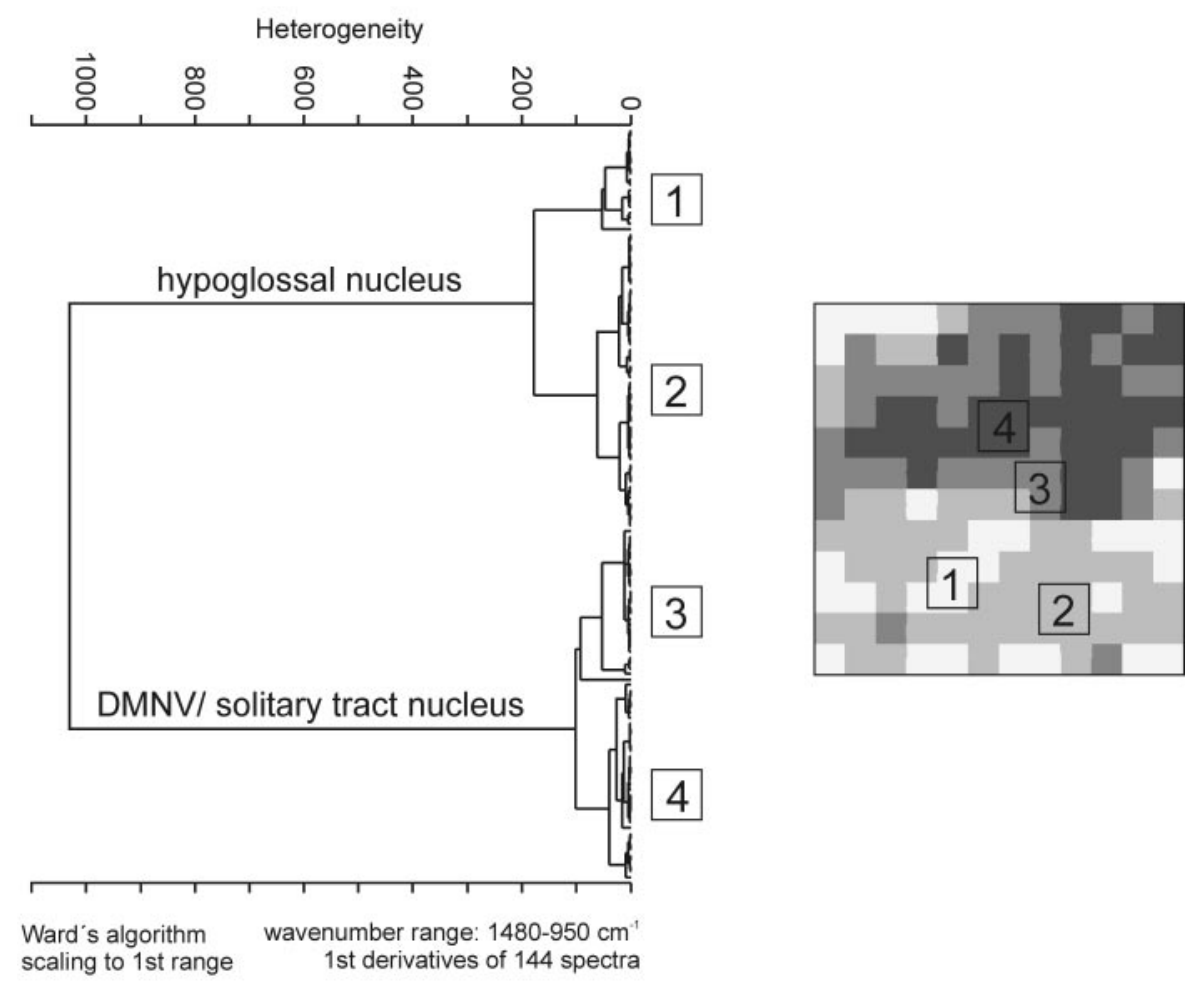

B

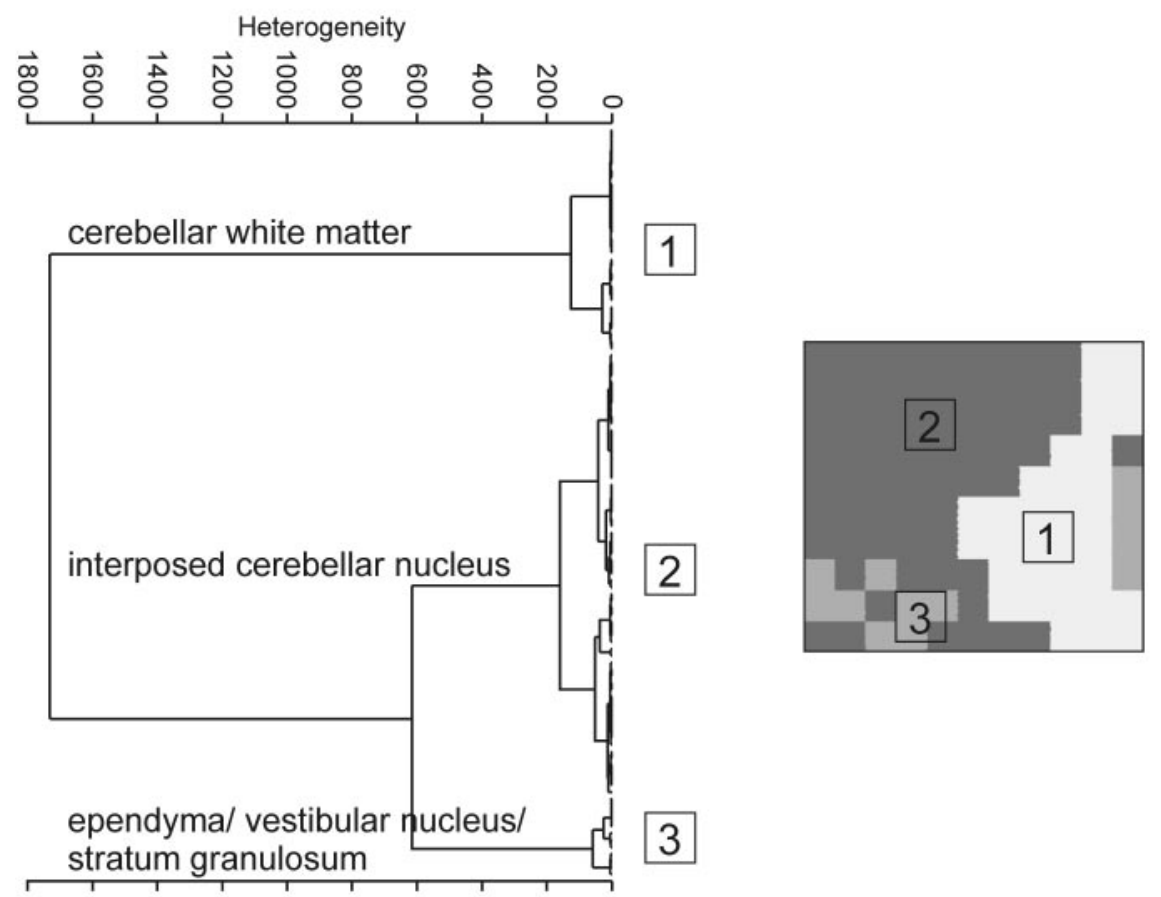

Figure 4. Results of cluster analyses for tissue structure identification displayed as dendrograms and pattern-based IR images. Results obtained for one individual are shown as an example. Each pixel in an image corresponds to one spectrum of the mapping data set. All spectra from one class are displayed in the same shade of gray; classes are numbered. After these cluster analyses, spectra of specific nuclei could be isolated from the data sets. $A$, Separation of spectra from plane 1 . Spectra from the DMNV and SolN appear as one spectral class (DMNV/SolN), which is clearly separated from spectra of the HypN. Two subclasses of each structure are displayed in the corresponding image. $B$, Spectra from detailed measurement of an area in plane 2. Gray and white matter structures are separated, and the spectra of IntN form a subcluster within the gray matter. Cluster analyses were performed over the frequency range $1480-950 \mathrm{~cm}^{-1}$, with first derivatives of the complete data sets obtained from the detailed measurements.
Ward's algorithm wavenumber range: $1480-950 \mathrm{~cm}^{-1}$ scaling to 1 st range 1 st derivatives of 110 spectra lin and Paxinos, 1997), measurements at $50 \mu \mathrm{m}$ spatial resolution were performed for specific nuclei in both coronal planes (see areas indicated in Fig. 3). In the images of plane 1, the HypN could be separated easily from the nuclei located more dorsally, such as the DMNV and SolN. The protein/lipid ratios of the
DMNV and parts of the SolN (central and/or intermediate part) were very similar. Multivariate cluster analyses were performed with all spectra from each detailed measurement in planes 1 and 2. New, cluster analysis-based maps were then reconstructed as shown in Figure 4. A comparison with the topology in the 
Table 2. Numbers of spectra per brain structure for different stages of infection as obtained from the data sets by cluster analysis from normal and infected animals

\begin{tabular}{|c|c|c|c|c|c|c|}
\hline \multirow{2}{*}{$\begin{array}{l}\text { Brain structure } \\
\text { (nucleus) }\end{array}$} & \multicolumn{2}{|c|}{ DMNV/SolN } & \multicolumn{2}{|c|}{ HypN } & \multicolumn{2}{|c|}{ IntN } \\
\hline & $\mathrm{N}$ & $\mathrm{S}$ & $\mathrm{N}$ & $\mathrm{S}$ & $\mathrm{N}$ & $\mathrm{S}$ \\
\hline Terminal stage & 178 & 229 & 223 & 210 & 364 & 362 \\
\hline 120 d.p.i. & 107 & 119 & 122 & 184 & 335 & 319 \\
\hline 90 d.p.i. & 94 & 122 & 233 & 228 & 397 & 403 \\
\hline
\end{tabular}

N, Normal; S, infected.

protein/lipid maps (Fig. $3 A$, grid) revealed that cluster analysis of all spectra obtained from plane 1 for each hamster had produced two major classes with the spectra of the HypN and DMNV/SolN, respectively (Fig. 4A). Furthermore, each cluster contained two spectral subclasses $(1,2$ and 3,4$)$ as displayed in the dendrogram of Figure $4 A$. Generally, the subclasses could not be assigned to particular histological structures, with the exception of two spectra in class 3 of Figure $4 A$, which were identified as stemming from the central canal. Hierarchical clustering of the spectra from the detailed measurements in plane 2 (Fig. 4B) showed separation of cerebellar white matter (cluster 1) from those of the adjacent IntN spectra (cluster 2) and also from spectra of other gray matter structures (cluster 3). Spectra from the latter belonged to the cerebellar cortex (stratum granulosum) and to medullar nuclei, such as the vestibular nucleus (Fig. 4B, cluster 3 ). The results of cluster analyses performed in the same way with the 24 different brain samples were very similar to the examples shown in Figure 4.

To directly compare spectral features in scrapie-affected brains with those of the controls, all single spectra of the DMNV/SolN and of the HypN from the plane 1 data sets and of IntN from the plane 2 data sets were extracted for all 24 individuals. Extraction was performed on the basis of the classification results shown in Figure 4. In this way, spectra from identical structures in healthy and diseased brains could be collated. The numbers of spectra per brain structure and infection stage are listed in Table 2.

\section{Comparison of average spectra}

In Figure $5 A$, normalized first derivatives of the average spectra of the DMNV/SolN, HypN, and IntN from terminally diseased and control hamsters are shown in the spectral region between 1300 and $1000 \mathrm{~cm}^{-1}$. First derivatives were calculated to enhance resolution of the spectral bands and to minimize slight baseline variations in the spectra. All peak maxima of the original absorption spectra appear as zero crossings in the first derivatives. It should be noted that the SDs in the groups of control animals were smaller than in those of the infected animals. The spectral region shown in Figure $5 A$ contains a number of distinct spectral variations of the infected tissue. Differences in the spectra can be found at characteristic positions between 1300 and $1000 \mathrm{~cm}^{-1}$, where various types of molecules, such as carbohydrates, lipids, and nucleic acids, contribute to the spectral profile (Fig. $5 A$ ). The spectral alterations are caused by changes in the absorption bands from a number of different functional groups present in these molecules. Between 1200 and $1000 \mathrm{~cm}^{-1}$, spectral characteristics are dominated by $\mathrm{C}-\mathrm{O}-\mathrm{P}$ and $\mathrm{C}-\mathrm{O}-\mathrm{C}$ stretching vibrations of carbohydrates and lipids. For example, the differences at 1170 $\mathrm{cm}^{-1}$ (antisymmetric $\mathrm{C}-\mathrm{O}-\mathrm{C}$ stretching vibration) hint at compositional and structural changes of molecules containing $\mathrm{C}-\mathrm{O}-\mathrm{C}$ functional groups. Changes of the absorbance band
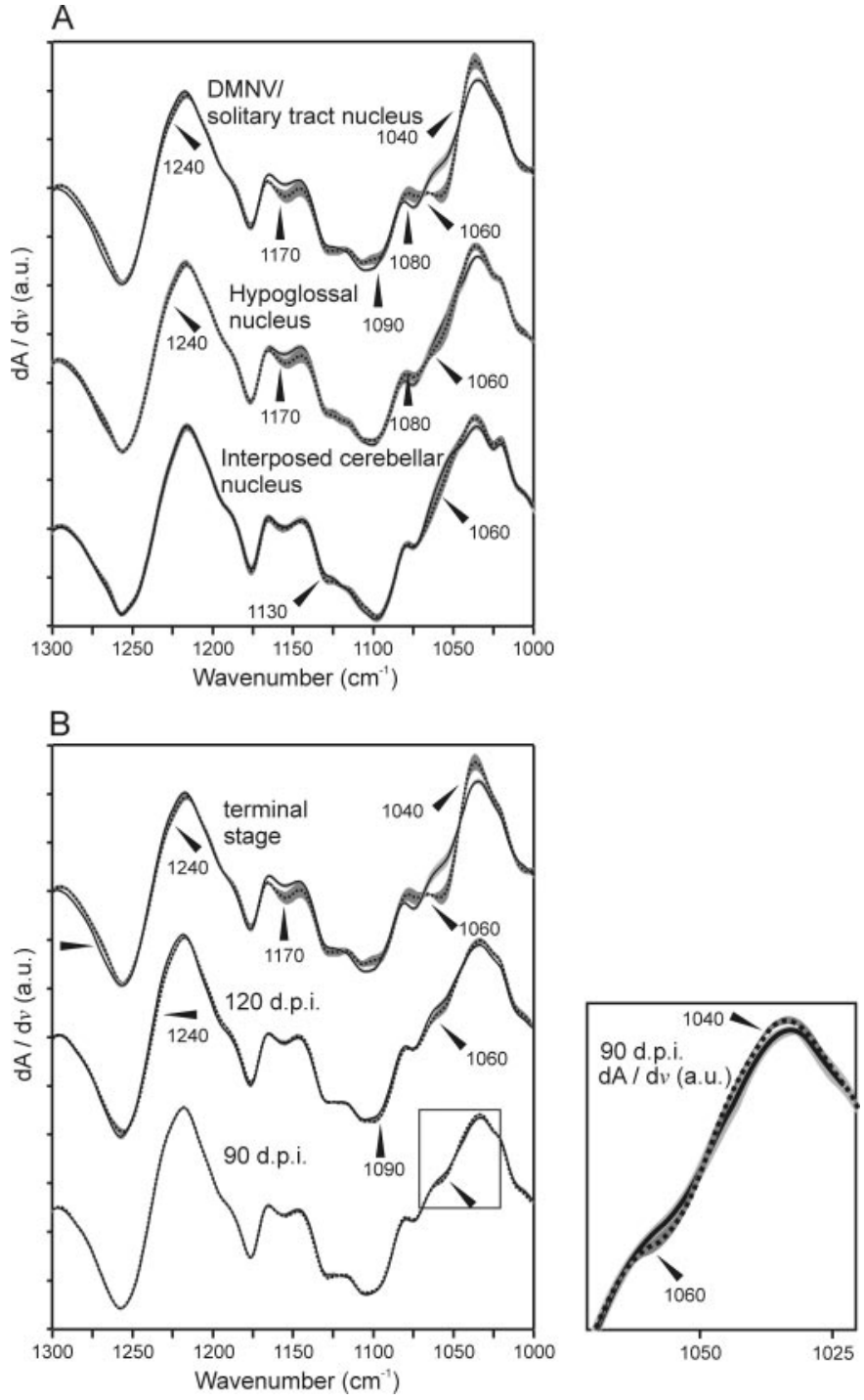

Figure 5. Normalized first derivatives of average spectra, each obtained from single spectra of four scrapie-infected hamsters (dashed lines) and four control hamsters (solid lines) with their SDs (dark, infected; light, normal controls) displayed over the frequency range $1300-1000 \mathrm{~cm}^{-1}$. Arrowheads indicate differences between spectra. All maxima appear as zero crossings. $A$, Average spectra of DMNV/SolN, HypN, and IntN at the terminal stage of the disease. $B$, Average spectra of DMNV/SolN at three stages of the disease. The inset displays the spectral region containing differences between the averages at 90 d.p.i.

resulting from the symmetric $\mathrm{P}=\mathrm{O}$ stretching vibration of $\mathrm{PO}_{2}{ }^{-}$groups of nucleic acids and phospholipids located at $\sim 1080 \mathrm{~cm}^{-1}$ (Liquier and Taillandier, 1996) were also observed. At $\sim 1240 \mathrm{~cm}^{-1}$, the width of the peak of the antisymmetric $\mathrm{P}=\mathrm{O}$ stretching vibration is slightly diminished in the DMNV/SolN and HypN of the infected samples. The prominent changes in band shape at $\sim 1060$ and $1040 \mathrm{~cm}^{-1}$ are probably caused by alterations in carbohydrates, because absorption bands at these frequencies can be assigned to complex sugar ring vibrations of these molecules (Parker, 1983). In the average spectra of the IntN, the spectral differences between infected and control hamsters are almost confined to this band (Fig. 5A).

Figure $5 B$ displays spectral differences between infected and control brains for average spectra of the DMNV/SolN at the 


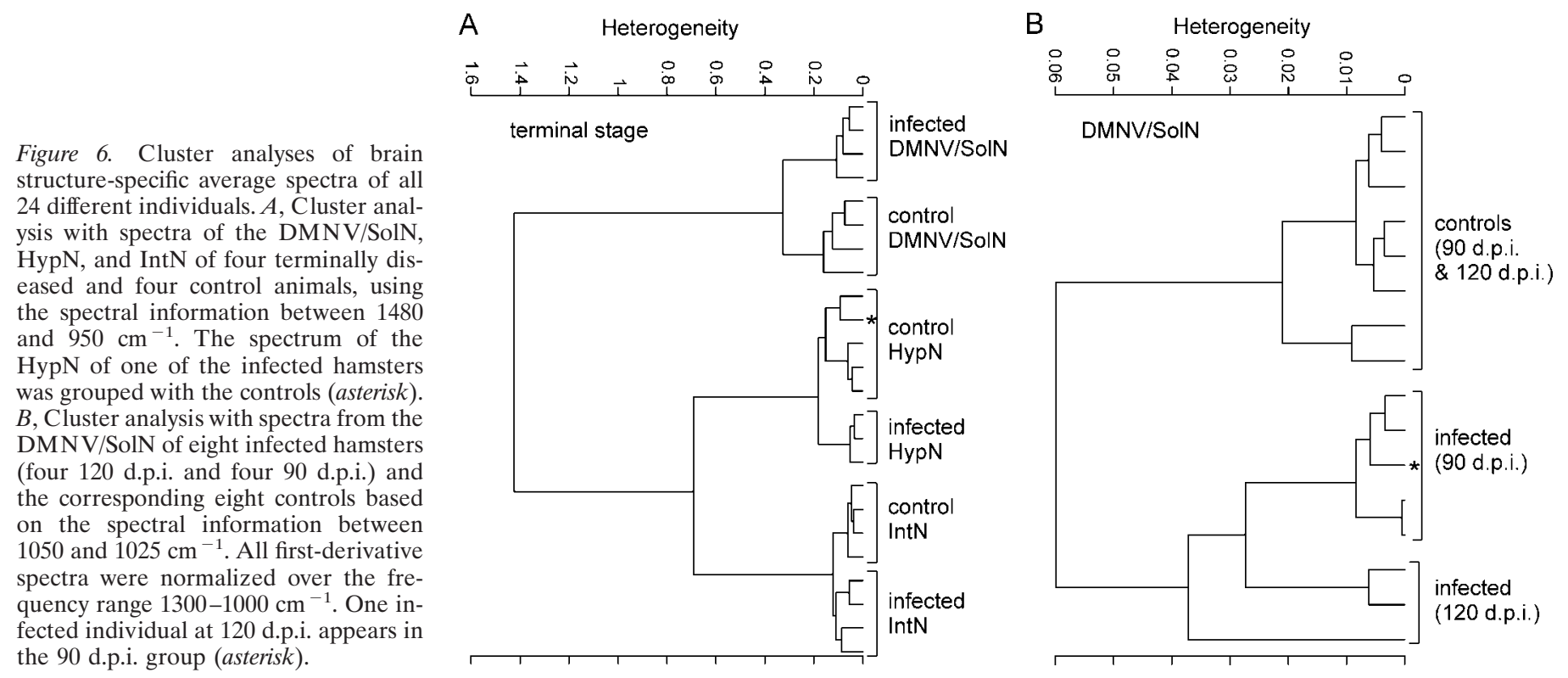

three different disease stages. In the control groups, the spectra of the DMNV/SolN are almost identical at all three stages (Fig. 5B, solid lines with light SD). In contrast, the average spectra of the diseased samples (Fig. 5B, dotted lines with dark SD) differ systematically between the investigated time points after infection. Variations in the spectra obtained from scrapie-infected DMNV/ SolN are most prominent in the terminal stage. In the region between 1060 and $1040 \mathrm{~cm}^{-1}$, the differences between healthy and infected sample spectra can be traced back even to 90 d.p.i. (Fig. 5B). They are small at 90 d.p.i. but clearly progress toward the terminal stage until a pronounced shoulder appears at $\sim 1050$ $\mathrm{cm}^{-1}$.

\section{Hierarchical clustering of spectra from diseased and control tissue}

For the objective comparison of structure-specific spectra from each investigated individual, a multivariate method was used. Average spectra for each nucleus and each individual in the terminal stage of scrapie and the controls were subjected to cluster analysis, using the information contained in the frequency range $1480-950 \mathrm{~cm}^{-1}$. Figure $6 A$ shows the result of this cluster analysis as a dendrogram. A clear separation of the different nuclei (DMNV/SolN, HypN, and IntN) can be observed (Fig. $6 A$ ). Within these structure-specific classes, spectra from infected and control tissue are separated. One HypN spectrum from the infected individuals was misclassified and grouped together with the HypN spectra from the controls. The disease-induced spectral differences in the DMNV/SolN at the earlier disease stages (120 and 90 d.p.i.) were also investigated on the level of structurespecific average spectra of the individuals. Figure $6 B$ shows the result of a cluster analysis of DMNV/SolN spectra of the infected animals at 90 and 120 d.p.i. and the corresponding controls. The spectra were normalized over the range $1300-1000 \mathrm{~cm}^{-1}$, and the region $1050-1025 \mathrm{~cm}^{-1}$ was used as input for cluster analysis. All spectra from the mock-infected controls of both stages appear as one group in the dendrogram, whereas another group is formed by the spectra from the infected animals. The spectra of 90 d.p.i. appear as one subgroup that also contains the spectrum of one of the 120 d.p.i. hamsters.

\section{ANN classification of single spectra from diseased and control tissue}

As a classification approach for single-point spectra, ANN analysis was applied to all mapping data sets. A feature extraction method based on univariate $F$ values was used to identify spectral features that differed significantly between spectra of diseased and control DMNV/SolN, HypN, and IntN at each of the three infection stages. On the basis of these features, networks were trained and used for identification of independent test spectra as described in Materials and Methods. The classification result for each spectrum was compared with the scrapie/control status of the corresponding tissue sample. The numbers of correctly classified test spectra were used to determine identification accuracy (Table 3 ). The numbers of selected data points for network training differed for the structures and infection stages and are also given in Table 3. Table 3 shows an increase of the identification accuracy of the networks with incubation time. At 90 d.p.i., the majority of single spectra from all investigated structures were still classified correctly by the ANN analyses.

\section{PrP ${ }^{\mathrm{Sc}}$ immunocytochemistry}

Cryosections adjacent to those investigated by FTIR microspectroscopy were stained with $\mathrm{mAb} 3 \mathrm{~F} 4$, which binds to the prion protein. As was shown previously, the pathological isoform $\mathrm{PrP}^{\mathrm{Sc}}$ can be distinguished from the cellular isoform $\operatorname{PrP}^{\mathrm{C}}$ by its morphological appearance (Beekes et al., 1998; McBride et al., 2001). The amount of $\mathrm{PrP}^{\mathrm{Sc}}$ detected at each time point varied between individuals. At the terminal stage, massive deposits of $\operatorname{PrP}^{\mathrm{Sc}}$ were found for the DMNV/SolN (Fig. 7A), HypN, and IntN. No PrP $\mathrm{P}^{\mathrm{Sc}}$ was detected in specimens from normal control animals (Fig. 7B). At 120 d.p.i., the accumulations were moderate in all structures and could be distinguished as single granules in the DMNV/ SolN, IntN (Fig. 7C), and HypN (Fig. 7D). Deposition was more pronounced in the DMNV/SolN than in the HypN and IntN. At 90 d.p.i., $\operatorname{PrP}^{\mathrm{Sc}}$ was undetected in the IntN and HypN. Some single neurons in the DMNV/SolN displayed small granular accumulations of the protein along their surface at this stage (Fig. $7 E)$. 


\begin{tabular}{|c|c|c|c|c|c|c|}
\hline \multirow{2}{*}{$\begin{array}{l}\text { Brain structure } \\
\text { (nucleus) }\end{array}$} & \multicolumn{2}{|c|}{ DMNV/SolN } & \multicolumn{2}{|l|}{ HypN } & \multicolumn{2}{|l|}{ IntN } \\
\hline & $\mathrm{N}$ & S & $\mathrm{N}$ & S & $\mathrm{N}$ & $\mathrm{S}$ \\
\hline Terminal stage & $\begin{array}{l}100 \% \\
{[100]}\end{array}$ & $100 \%$ & $\begin{array}{l}100 \% \\
{[100]}\end{array}$ & $99.0 \%$ & $\begin{array}{l}98.3 \% \\
{[100]}\end{array}$ & $98.6 \%$ \\
\hline 120 d.p.i. & $\begin{array}{l}93.5 \% \\
{[50]}\end{array}$ & $95.8 \%$ & $\begin{array}{l}81.1 \% \\
{[50]}\end{array}$ & $86.9 \%$ & $\begin{array}{l}88.6 \% \\
{[50]}\end{array}$ & $85.6 \%$ \\
\hline 90 d.p.i. & $\begin{array}{l}70.2 \% \\
{[16]}\end{array}$ & $77.0 \%$ & $\begin{array}{l}80.3 \% \\
{[16]}\end{array}$ & $79.3 \%$ & $\begin{array}{l}76.0 \% \\
{[16]}\end{array}$ & $74.7 \%$ \\
\hline
\end{tabular}

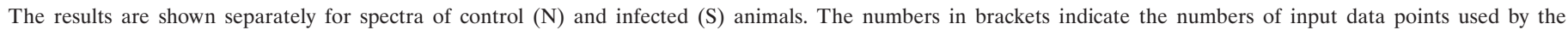
corresponding ANN.

\section{DISCUSSION}

The results of this work demonstrate that changes in the IR spectra of the DMNV/SolN, HypN, and IntN can be used to distinguish scrapie-infected from uninfected tissue. The procedure applied initially included identification of structure-specific spectra from various nuclei in the brain by cluster analysis and IR imaging, followed by extraction of these spectra for comparative purposes. The necessity of collating identical brain structures when comparing the spectral characteristics of normal tissue with those of disease-affected tissue can be illustrated by the comparison of average spectra from infected and control animals (Fig. 5). As proven by cluster analysis, the disease-related spectral variations were indeed smaller than the spectral differences between distinct histological structures (Fig. 6A).

Because one or more absorption bands correspond to a functional group characteristic for a class of molecules rather than for one specific compound, and because spectral variations occur in a number of different absorption bands, it can be concluded that superposition of multimolecular information is the basis of the disease-specific spectral changes. On the molecular level, the observed spectral differences are consistent with phenotypic features found, for example, in ultrastructural studies that occur in early stages of the disease (Liberski et al., 1989; Jeffrey et al., 1995). These are, for example, microvacuolation, membrane proliferation, and structural and functional damage of mitochondria (Choi et al., 1998), in which changes in the content of phospholipids take place, as was suggested in this study by variations of the antisymmetric and symmetric $\mathrm{P}=\mathrm{O}$ stretching vibrations of the polar head groups of the lipid and altered absorption of $\mathrm{C}-\mathrm{O}-\mathrm{C}$ stretching vibrations. Changes in absorption bands containing contributions from nucleic acids (ribose skeletal $\mathrm{C}-\mathrm{O}-\mathrm{C}$ and $\mathrm{C}-\mathrm{C}$ stretching vibrations and $\mathrm{P}=\mathrm{O}$ stretching vibrations) may also indicate changes in the DNA/RNA ratio caused, for example, by DNA decomposition during apoptosis (Fairbairn et al., 1994; Lucassen et al., 1995) and/or a changed RNA content as the result of upregulation or downregulation of genes (Diedrich et al., 1993; Riemer et al., 2000).

The first change of spectral features that could be observed for diseased DMNV/SolN at 90 d.p.i. and that remained prominent until the terminal stage was a change in band shape between 1060 and $1040 \mathrm{~cm}^{-1}$ (Fig. 5B). This indicates an altered absorption of ring vibrations of carbohydrates that could be assigned to the sugar moieties of nucleic acids, to changed content of metabolic sugar molecules in the cells, such as glucose, or to other events that have not yet been described. Glucose metabolism is known to change in intracerebrally infected hamsters (Gregoire et al., 1983) and in fatal familial insomnia, a human TSE (Cortelli et al., 1997).
Little is known about the extent of these effects and which of them prevail. The above-mentioned spectral change at $\sim 1050$ $\mathrm{cm}^{-1}$ is one of the features that was common to all investigated brain structures. However, this change was not equally expressed in all nuclei (Fig. 5A). These quantitative differences of spectral changes occurring in all structures, as well as qualitative absorbance differences (pointing to different molecular events taking place), were observed between the different nuclei. These findings are also reflected by the different heterogeneities between infected and control tissue spectra within each tissue structure in the dendrogram of Figure $6 A$.

The findings that spectral differences are clearly progressing (i.e., becoming more and more prominent) during the course of the disease in the average spectra (Fig. $5 B$ ) and that identification accuracy of ANN analyses increases with incubation time (Table 3 ) suggest that in the data sets obtained from the infected animals in earlier stages, a number of misclassified single spectra did not exhibit spectral features sufficient to make them distinguishable from those of control animals. This is similar to the well known spread of the histopathological features in scrapie, such as $\mathrm{PrP}^{\mathrm{Sc}}$ deposition. Early in pathogenesis, this pathological form of the prion protein in the DMNV is confined to individual neurons (van Keulen et al., 2000), whereas in the terminal stage, it can be detected in and around almost every neuron of a nucleus. Disease-specific features in the IR spectra were very pronounced in the terminal stage, so that almost every point spectrum obtained from a nucleus during a mapping measurement (i.e., every spot in the mapped area) was classified correctly as "diseased" by the method applied in this study (Table 3 ). As could be shown by ANN analysis, even at 90 d.p.i., most of the spectra obtained from the DMNV/SolN, HypN, and IntN still exhibited disease-specific IR spectral features.

When average spectra were calculated for each group of infected and control animals, the SDs of the spectra were smaller for the control animals than for the scrapie-infected animals. This finding indicates that individual parameters seem to determine how rapidly and to what extent molecular changes have developed at a certain stage of the disease. Assuming that biochemical changes begin to be visible in the IR spectra of a specific cell type, it would be interesting to identify these histological substructures or cell types. Systematic comparisons of the spectra on the level of cell populations within the nuclei could then facilitate the detection of scrapie-specific features (such as events confined to neurons or proliferation processes in astrocytes) (Eklund et al., 1963) early in pathogenesis in situ without using immunocytochemistry but by comparing spectra of the identical cell populations of the DMNV/SolN in infected and control brains. Image 


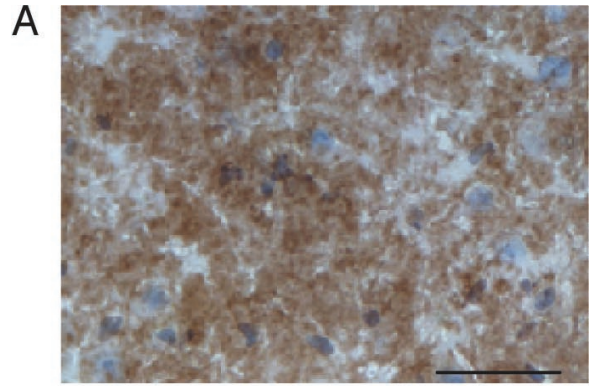

B

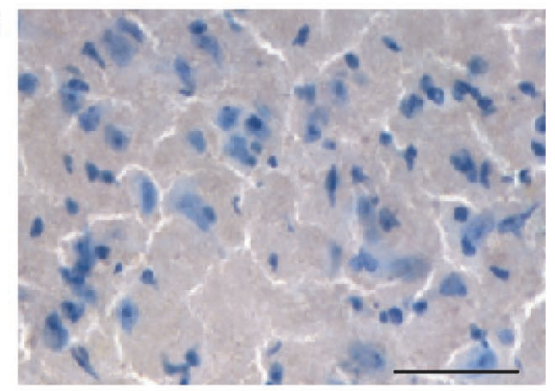

C

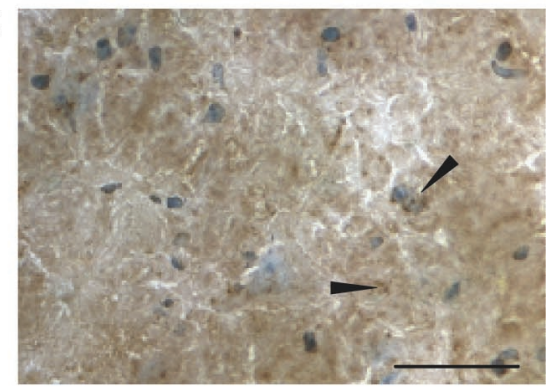

D

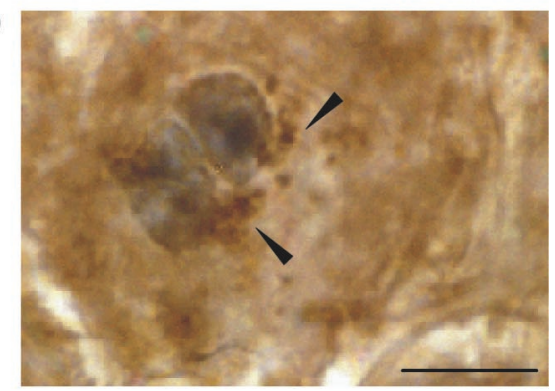

E

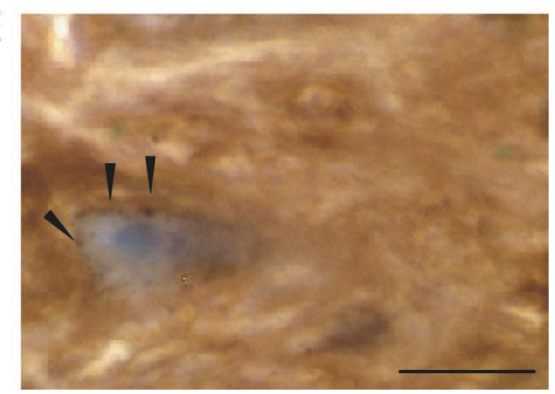

Figure 7. $\quad \operatorname{PrP}^{\mathrm{Sc}}$ immunolabeling in sections adjacent to those investigated by FTIR microspectroscopy. $\operatorname{PrP}^{\mathrm{Sc}}$ characteristically presents as granular accumulations of varying sizes. $A$, Massive $\mathrm{PrP}^{\mathrm{Sc}}$ deposition in the DMNV/SolN at the terminal stage. $B$, The DMNV/SolN of a mockinfected control. $C$, An IntN at 120 d.p.i. D, A HypN at 120 d.p.i. E, A DMNV/SolN at 90 d.p.i. Arrowheads point to islets of granular accumulations. Scale bars: $A-C, 40 \mu \mathrm{m} ; D, E, 10 \mu \mathrm{m}$. reconstruction based on the results of cluster analyses revealed that the two subclusters within the two major spectral classes, DMNV/SolN and HypN (Fig. 4A), correspond to relatively small spots (comprising only a few spectra) that are equally distributed in the two nuclei. What is the histological basis of these subclusters? This question can only be addressed by acquiring spectra from single cells in scrapie-affected tissue with high spatial resolution by applying an IR synchrotron source (Jamin et al., 1998).

From histological studies of the $263 \mathrm{~K}$ scrapie model in hamsters, it is known that the DMNV and the SolN are the first regions in the brain in which an orally induced scrapie infection can be detected by immunostaining of $\mathrm{PrP}^{\mathrm{Sc}}$ deposits in paraffin sections. Much later in the disease process, other nuclei, among them HypN and IntN, are affected (Beekes et al., 1998). The results of our study match these observations: The comparison of average spectra from the DMNV/SolN in scrapie-infected and control animals revealed differences already at 90 d.p.i., whereas changes in the HypN and IntN could not be detected earlier than 120 d.p.i. (data not shown). Furthermore, differences between average spectra in the terminal stage were more pronounced in the DMNV/SolN than in the HypN and IntN (Fig. 5A). These findings are also in good accordance with results from immunostaining of the adjacent cryosections in the terminal stage and at 120 d.p.i. and with studies at early disease stages (McBride et al., 2001), when $\operatorname{PrP}^{\mathrm{Sc}}$ deposition was always heavier in the DMNV and SolN than in the HypN. The extent and local detectability of the IR spectral variations (Fig. 5) obviously concur with the known sequence of $\operatorname{PrP}^{\mathrm{Sc}}$ deposition. Thus, IR spectral changes provide a new biophysical parameter based on molecular markers that indicate the spread of scrapie pathology in the brain starting in the DMNV and SolN.

It is important to note that in contrast to immunocytochemistry, early detection of TSE by IR spectroscopy is not based on the spectral features of $\mathrm{PrP}^{\mathrm{Sc}}$ but rather on changes in a number of different molecules. The spectral data indicate that a diseasespecific change of carbohydrates, nucleic acids, and membrane constituents exists early in pathogenesis. The fingerprint-like nature of the scrapie-induced spectral variations greatly diminishes the probability of an identical multivariate pattern being observed in a different disease, such as pseudorabies, herpes simplex type 1 , or reovirus serotype 3 (isolate T3C9) infections, which were shown to affect the DMNV in specific animal models (Card et al., 1990; Krinke and Dietrich, 1990; Morrison et al., 1991). However, to further assess the specificity of the IR method, other infections of the CNS of nonexperimental donors will have to be investigated in future studies. The potential sensitivity of the IR method compared with that of very sensitive $\mathrm{PrP}^{\mathrm{Sc}}$ staining methods, especially with that of the paraffinembedded tissue blot (Schultz-Schaeffer et al., 2000), can be discussed only when FTIR spectroscopic analysis on the singlecell level (as was discussed above) is established. The advantage of the IR spectroscopic method is that only frozen sections without any fixation or staining are required. Fast computerized methods can be used for identification of the diseased tissue, providing a method for the investigation of TSE pathogenesis that can also be developed into a rapid postmortem diagnostic screening method. Because TSE-related cell or tissue damage is most prominent in the brain and spinal cord, we focused on the IR investigation of samples from the CNS. Future IR experiments will also address peripheral organs involved in TSE pathogenesis, such as the peripheral nervous system and the lymphoreticular 
system, along with samples suited for in vivo testing, such as blood or CSF.

\section{REFERENCES}

Beekes M, McBride PA, Baldauf E (1998) Cerebral targeting indicates vagal spread of infection in hamsters fed with scrapie. J Gen Virol 79:601-607.

Card JP, Rinaman L, Schwaber JS, Miselis RR, Whealy ME, Robbin AK, Enquist LW (1990) Neurotropic properties of pseudorabies virus: uptake and transneuronal passage in the rat central nervous system. J Neurosci 10:1974-1994.

Choi S-I, Ju W-K, Choi E-K, Kim J, Lea H-Z, Carp RI, Wisniewski HM, Kim Y-S (1998) Mitochondrial dysfunction induced by oxidative stress in the brains of hamsters infected with the $263 \mathrm{~K}$ scrapie agent. Acta Neuropathol 96:279-286.

Cortelli P, Perani D, Parchi P, Grassi F, Montagna P, DeMartin M, Castellani R, Tinuper P, Gambetti P, Lugaresi E, Fazio F (1997) Cerebral metabolism in fatal familial insomnia. Neurology 49:126-133.

Diedrich JF, Carp RI, Haase AT (1993) Increased expression of heat shock protein, transferrin, and $\beta$-microglobulin in astrocytes during scrapie. Microb Pathog 15:1-6.

Eklund CM, Hadlow WJ, Kennedy RC (1963) Some properties of the scrapie agent and its behaviour in mice. Proc Soc Exp Biol Med 112:974-979.

Fairbairn DW, Carnahan KG, Thwaits RN, Grigsby RV, Holyoak GR, O'Neill KL (1994) Detection of apoptosis induced DNA cleavage in scrapie-infected sheep brain. FEMS Microbiol Lett 115:341-346.

Franklin KB, Paxinos G (1997) The mouse brain in stereotaxic coordinates. San Diego: Academic.

Goodacre R (2000) Applications of artificial neural networks to the analysis of multivariate data. In: Intelligent data analysis in science (Cartwright H, ed), pp 123-152. Oxford: Oxford UP.

Gregoire N, Gorde-Durand J, Bouras C, Salamon G, Bert J (1983) Cerebral glucose utilization: local changes after microinoculation of scrapie agent in hamster. Neurosci Lett 36:181-187.

Hedge RS, Tremblay P, Groth D, DeArmond SJ, Prusiner SB, Lingappa VR (1999) Transmissible and genetic prion diseases share a common pathway of neurodegeneration. Nature 402:822-826.

Helm D, Labischinski H, Schallehn G, Naumann D (1991) Classification and identification of bacteria by Fourier-transform infrared spectroscopy. J Gen Microbiol 137:69-79.

Jamin N, Dumas P, Moncuit J, Fridman W-H, Teillaud J-L, Carr GL, Williams GP (1998) Highly resolved chemical imaging of living cells by using synchrotron infrared microspectrometry. Proc Natl Acad Sci USA 95:4837-4840.

Jeffrey M, Goodbrand IA, Goodsir CM (1995) Pathology of transmissible spongiform encephalopathies with special emphasis on ultrastructures. Micron 26:277-298.

Kascsak RJ, Rubenstein R, Merz PA, Tonna-DeMasi M, Fersko R, Carp RI, Wisniewski HM, Diringer H (1987) Mouse polyclonal and monoclonal antibody to scrapie-associated fibril proteins. J Virol 61:3688-3693.

Kidder LH, Kalasinsky VF, Luke JL, Levin IW, Lewis EN (1997) Visualization of silicone gel in human breast tissue using new infrared imaging spectroscopy. Nat Med 3:235-237.

Kneipp J, Lasch P, Baldauf E, Beekes M, Naumann D (2000) Detection of pathological molecular alterations in scrapie-infected hamster brain by Fourier transform infrared (FT-IR) spectroscopy. Biochim Biophys Acta 1501:189-199.

Knigge KM, Joseph SA (1968) A stereotaxic atlas of the brain of the golden hamster. In: The golden hamster: its biology and use in medical research (Hoffman RA, Robinson PF, Magalhaes H, eds). Ames, Iowa: Iowa State UP

Krinke GJ, Dietrich FM (1990) Transneuronal spread of intraperitoneally administered herpes simplex virus type 1 from the abdomen via the vagus nerve to the brains of mice. J Comp Pathol 103:301-306.

Lasch P, Naumann D (1998) FT-IR microspectroscopic imaging of human carcinoma thin sections based on pattern recognition techniques. Cell Mol Biol 44:189-202.

Lewis EN, Gorbach AM, Marcott C, Levin IW (1996) High-fidelity Fourier transform infrared spectroscopic imaging of primate brain tissue. Appl Spectrosc 50:263-269.

Liberski PP, Asher DM, Yanagihara R, Gibbs Jr CJ, Gajdusek DC (1989) Serial ultrastructural studies of scrapie in hamsters. J Comp Pathol 101:429-442.

Liquier J, Taillandier R (1996) Infrared spectroscopy of nucleic acids. In: Infrared spectroscopy of biological molecules (Mantsch $\mathrm{HH}$, Chapman D, eds), pp 131-158. New York: Wiley.

Lucassen PJ, Williams A, Chung WCJ, Fraser H (1995) Detection of apoptosis in murine scrapie. Neurosci Lett 198:185-188.

McBride PA, Schulz-Schaeffer W, Donaldson M, Bruce ME, Diringer H, Kretzschmar H, Beekes M (2001) Early spread of scrapie from the gastrointestinal tract to the central nervous system involves autonomic fibres of the splanchnic and vagus nerves. J Virol 75:9320-9327.

Morrison LA, Sidman RL, Fields NB (1991) Direct spread of reovirus from the intestinal lumen to the central nervous system through vagal autonomic nerve fibers. Proc Natl Acad Sci USA 88:3852-3856.

Parker FS (1983) Applications of infrared, Raman, and resonance Raman spectroscopy in biochemistry. New York: Plenum.

Prusiner SB, Scott MR, DeArmond SJ, Cohen FE (1998) Prion protein biology. Cell 93:337-348

Riedmiller M, Braun H (1993) A direct adaptive method for faster backpropagation learning: the RPROP algorithm. In: IEEE International Conference on Neural Networks (ICNN), Vol 2, pp 586-591. Piscataway, NJ: IEEE.

Riemer C, Queck I, Simon D, Kurth R, Baier M (2000) Identification of upregulated genes in scrapie-infected brain tissue. $\mathrm{J}$ Virol 74:10245-10248.

Schultz-Schaeffer WJ, Tschöke S, Kranefuss N, Dröse W, Hause-Reitner D, Giese A, Groschup MH, Kretzschmar HA (2000) The paraffinembedded tissue blot detects PrPSc early in the incubation time in prion diseases. Am J Pathol 156:51-56.

Taraboulos A, Jendroska K, Serban D, Yang S-L, DeArmond SJ, Prusiner SB (1992) Regional mapping of prion proteins in brain. Proc Natl Acad Sci USA 89:7620-7624.

Udelhoven T, Naumann D, Schmitt J (2000) Development of a hierarchical classification system with artificial neural networks and FT-IR spectra for the identification of bacteria. Appl Spectrosc 54:1471-1479.

van Keulen LJM, Schreuder BEC, Vromans MEW, Langeveldt JPM Smits MA (2000) Pathogenesis of natural scrapie in sheep. Arch Virol Suppl 16:57-71.

Wetzel DL, LeVine SM (1999) Imaging molecular chemistry with infrared microscopy. Science 285:1224-1225. 